\title{
Acute Presentation of Massive Retrosternal Thyrotoxic Goitres*
}

\author{
Joshil V. Lodhia ${ }^{1}$, Thomas D. Christensen ${ }^{1,2}$, Ehab S. Bishay ${ }^{1}$, Maninder S. Kalkat ${ }^{1}$ \\ ${ }^{1}$ Dept. of Thoracic Surgery, Heart of England Foundation Trust, Birmingham, UK; ${ }^{2}$ Dept. of Cardiothoracic and Vascular Surgery \& \\ Institute of Clinical Medicine, Aarhus University Hospital, Aarhus, Denmark. \\ Email: joshil.lodhia@nhs.net
}

Received July 24 $4^{\text {th }}, 2013$; revised August 21 ${ }^{\text {st }}, 2013$; accepted September $4^{\text {th }}, 2013$

Copyright @ 2013 Joshil V. Lodhia et al. This is an open access article distributed under the Creative Commons Attribution License, which permits unrestricted use, distribution, and reproduction in any medium, provided the original work is properly cited.

\begin{abstract}
Approximately 5\% of goitres extend below the thoracic inlet and can potentially become life threatening due to compression of the airway and major vessels. Approximately $7 \%$ of these goitres which require surgical resection will need an additional sternotomy to deliver the intra-thoracic component. Massive retrosternal toxic goitres presenting acutely are rare and are described infrequently in literature. We hereby present two cases of massive retrosternal thyrotoxic goitres presenting with acute respiratory failure, requiring non-invasive ventilation, as well as significant head and neck venous compression. Surgery on the thyrotoxic patient with a goitre, even if not significantly enlarged, is associated with a high peri-operative mortality due to cardiac instability and hemorrhage. We discuss the challenges of surgical intervention in these patients with particular emphasis on the timing of surgery to relieve compressive symptoms and the time needed to achieve a euthyroid state. We also emphasize the need for meticulous hemostasis, use of a cell-saver, transfusion protocols, adjuncts to hemostasis, as well as careful monitoring and continuous adjustments to the coagulation profile.
\end{abstract}

Keywords: Perioperative Issues and Risk Analysis; Retro-Sternal Goitres; Surgical Management; Sternotomy

\section{Introduction}

Goitre is a common condition with the definition of an enlargement of thyroid tissue. The accepted definition of retrosternal goitres are extension of greater than $50 \%$ of the thyroid tissue into the mediastinum. In $5 \%$ there is retrosternal extension, which can become life threatening if there is a rapid enlargement or hemorrhage [1]. This diagnosis is most often made in the fifth or sixth decade of life and has a female predominance of 4:1 [1]. Very large retrosternal goitres above $800 \mathrm{~g}$ are rare and few are described in literature [1,2]. Due to the toxic nature and the compression of surrounding respiratory and vascular structures these large goiters pose a high risk for surgical intervention. We report two cases of large thyrotoxic retrosternal goitres (906 g and $1499 \mathrm{~g}$ ) with attention to their clinical presentation, imaging and surgical approach.

\footnotetext{
*Thomas D. Christensen has done lectures supported by AstraZeneca,
} Boehringer Ingelheim, Pfizer, and Takeda.

\section{Case Reports}

\subsection{Case One}

Case one is a 64-year-old female who presented with shortness of breath. She presented in a thyrotoxic state. Her free thyroxine level was $21.2 \mathrm{pmol} / \mathrm{L}(9.0$ - 19.0) and thyroid stimulating hormone $(\mathrm{TSH})<0.01 \mathrm{mU} / \mathrm{L}(0.4-$ 4.9). CXR and subsequent CT scan showed a large mediastinal mass compressing the brachiocephalic vein and trachea (Figure 1). Prior to surgery she was requiring biphasic positive airway pressure (BPAP) ventilation in the high dependency unit (HDU) for hypercapnic respiratory failure. She was treated with carbimazole for her thyrotoxicosis. The mass was removed through a transverse cervical and median sternotomy incisions. Despite hemostasis prior to closure the crater left a large surface area to bleed from for which she was returned to theatres for re-exploration. Following a short stay in the intensive care unit for re-expansion pulmonary edema the patient was returned to the ward and shortly discharged thereafter. The removed mass weighed $906 \mathrm{~g}$ and microscopic 


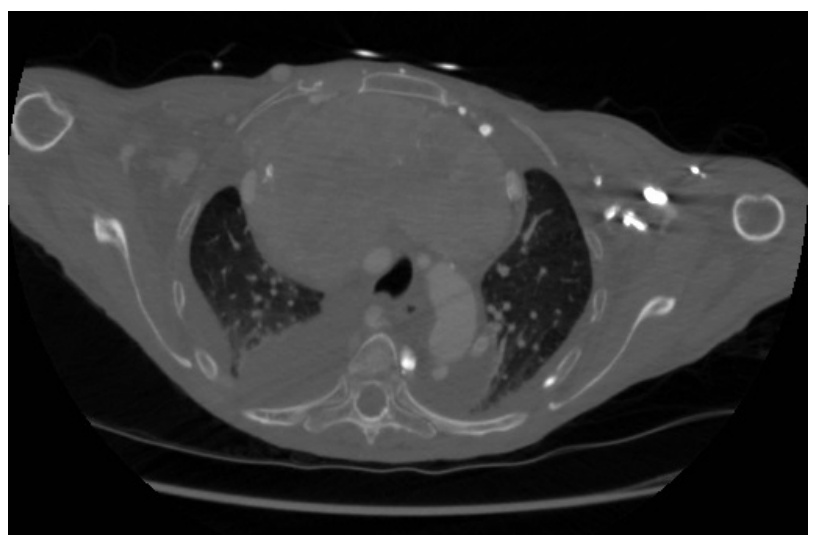

Figure 1. Pre-operative CT scan of case one (Level T3-T4). Note the compression of mediastinal structures such as the trachea and brachiocephalic vein.

findings were of a colloid goitre with no evidence of neoplasm.

\subsection{Case Two}

Case two was also a 64-year-old female who presented with shortness of breath and required BPAP on HDU. She had undergone a partial thyroidectomy 1986 and in 1999 was referred to a cardiothoracic surgeon for further resection. At this stage she declined surgical intervention. On her latest admission a CT scan showed a large multinodular retrosternal goitre (Figure 2). She was also found to have situs inversus with dextrocardia. Her thyroxine levels were 30.4 and TSH $<0.01$. The initial approach was via a cervical incision and a median sternotomy. Due to the extensive occupancy of the chest cavity by the mass a further left sided thoracotomy was performed. The difficulty in mobilizing the mass was that it compressed the heart and compromised venous return. Despite preoperative treatment with carbimazole and Lugol's iodine the thyroid mass was very vascular and there was significant blood loss. The total mass of the thyroid was $1499 \mathrm{~g}$. Microscopic findings were of a multi-nodular goitre with focal cystic changes and no evidence of malignancy. Post-operatively there was difficulty ensuring an adequate blood pressure and chest radiographs suggested re-expansion pulmonary edema. The patient died two days following the procedure due to ischemic hepatic insult, renal failure and subsequently multi-organ dysfunction.

\section{Discussion}

Multinodular goiters arises from the variable growth of individual nodules. These may follow a path of hemorrhage, degeneration, healing and fibrosis. One of the most common symptoms of compression from retrosternal goitres is dyspnea. Both our patients presented with these symptoms and given the slow growth of the mass and

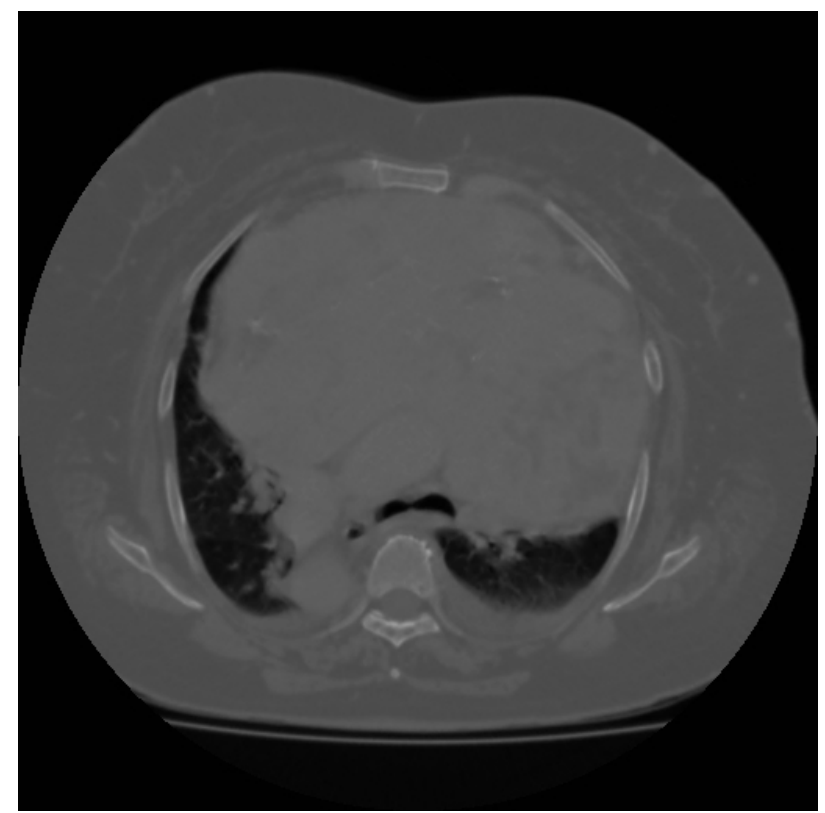

Figure 2. Pre-operative CT scan of case two (Level T4-T5). Notice the multi-nodularity of the retrosternal mass and the right sided position of the descending aorta.

typical age of these patients these symptoms are often mistaken for diagnoses such as chronic obstructive pulmonary disease or cardiac failure [3]. The presence of dyspnea and history of prior thyroid resection, as in case two, should alert the physician of a recurrent goitre. The interval between first surgical resection and recurrence is approximately 18 years [1]. The second case presented here was initially mistaken for heart failure following a chest radiograph. Tracheal deviation is the most common radiological sign and is present in approximately $75 \%$ of cases, including our two patients [1,4].

Non-surgical treatment by means of radioactive iodine and a thionamide is rarely successful. $1 \%-11 \%$ of resections require a sternotomy [2,5]. Factors found to increase the need for an extra-cervical approach are malignancy, weight of thyroid tissue and extension to the carina [1,2].

Thyrotoxic goitres have been operated on since their description in the 1830s. These goitres are highly vascular and pose a significant risk of hemorrhage in the perioperative period. High mortality rates did not decrease until the use of pre-operative iodine and subsequently thionamides [6]. The use of iodine is hypothesized to increase colloid formation which in turn leads to thyroid enlargement and compression of local vasculature [7]. This swelling of the mass was noted in the second case and necessitated the need for multiple approaches. Ideally patients should be medically optimized with thionamides prior to surgery with the aim of ensuring a euthyroid state. Due to respiratory failure, our two patients did not have the luxury of time for medical optimization. 


\section{Summary}

The outcome of our second case could be explained by her increased comorbidities. She presented in extremis and required immediate care in the intensive care setting. This is to stress the gravity of managing massive thyrotoxic goitres.

Our recommendations are that these retrosternal goitres should be removed early due to the risk of compressive symptoms. Chest radiographs are a good first line investigation and CT should follow thereafter. Sternotomy should be considered in cases where there are suggestions of malignancy or a large thyroid volume. Hemorrhage should be anticipated and adequate preparations made. This should be by the means of administering thionamides as early as possible, the use of intra-operative cell salvage, monitoring coagulation during and after the procedure by the use of thromboelastography (TEG ${ }^{\circledR}$ or ROTEM ${ }^{\circledR}$ ) and correction with blood products and clotting factors.

\section{REFERENCES}

[1] M. G. Rugiu and M. Piemonte, "Surgical Approach to Retrosternal Goitre: Do We Still Need Sternotomy?” Acta Otorhinolaryngolocia Italica, Vol. 29, No. 6, 2009, pp. 331-338.
[2] M. L. White and G. M. Doherty, "Evidence-Based Surgical Management of Substernal Goitre,” World Journal of Surgery, Vol. 32, No. 7, 2008, pp. 1285-1300. doi:10.1007/s00268-008-9466-3

[3] M. Nervi, P. Lacconi, C. Spinelli, A. Janni and P. Miccoli, “Thyroid Carcinoma Is Intrathoracic Goiter," Langenbeck's Archive of Surgery, Vol. 383, No. 5, 1998, pp. 337339. doi:10.1007/s004230050144

[4] M. R. Katlic and H. C. Grillo, "Substernal Goitre: Analysis of 80 Patients from Massachusetts General Hospital," American Journal of Surgery, Vol. 149, No. 2, 1985, pp. 283-287. doi:10.1016/S0002-9610(85)80086-6

[5] D. Kilic, A. Findikcioglu, Y. Ekici, U. Alemdaroglu, K. Hekimoglu and A. Hatipoglu, "When Is Transthoracic Approach Indicated in Retrosternal Goitres?” Annals of Thoracic and Cardiovascular Surgery, Vol. 17, No. 3, 2011, pp. 250-253. doi:10.5761/atcs.oa.10.01541

[6] E. B. Astwood, "Treatment of Hyperthyroidism with Thiourea and Thiouracil," Journal of American Medical Association, Vol. 122, No. 2, 1943, pp. 78-81. doi:10.1001/jama.1943.02840190008003

[7] J. H. Marigold, A. K. Morgan, D. J. Earle, A. E. Young and D. N. Croft, "Lugol's Iodine: Its Effect on Thyroid Blood Flow in Patients with Thyrotoxicosis," British Journal of Surgery, Vol. 72, No. 1, 1985, pp. 45-47. doi:10.1002/bjs.1800720118 